Wojciech Wojdowski, Department of Mathematics, Technical University of Łódź, Al. Politechniki 11, 90-924 Łódź, Poland. e-mail:

wwoj@ck-sg.p.lodz.pl

\title{
CONTINUITY IN TERMS OF FUNCTIONAL CONVERGENCE
}

\begin{abstract}
The note presents a new approach to the notion of continuity of real function at a point. It is applied to obtain a characterization of continuity at a point with respect to *-topology (Hashimoto topology), density topology and $I$-density topology (Wilczyński topology). The latter is closely related to the definition of density point of measurable set formulated by W. Wilczyński in [8].
\end{abstract}

Let $f: \mathbb{R} \rightarrow \mathbb{R}$ be an arbitrary function. For any sequence $\left\{t_{n}\right\}_{n \in N}$ of real numbers decreasing to zero, with $\left(t_{1}<1\right)$, we define a sequence of functions $\left\{f_{t_{n}}\right\}_{n \in N}, f_{t_{n}}:[-1,1] \rightarrow \mathbb{R}$ in the following way

$$
f_{t_{n}}(x)=f\left(t_{n} \cdot x\right)
$$

The theorem given below presents a new point of view on the notion of continuity. It describes a surprising connection between a continuity of a function at the point and a convergence of an appropriate sequence of functions. We omit the proof since it follows immediately from Cauchy's and Heine's definitions of continuity of a function at a point.

Theorem 1. The following conditions are equivalent:

(1) $f$ is continuous (with respect to the natural topology on the domain and on the range) at 0 ,

(2) for every sequence $\left\{t_{n}\right\}_{n \in \mathbb{N}}$ decreasing to zero the sequence of functions $\left\{f_{t_{n}}\right\}_{n \in \mathbb{N}}$ converges to $f(0)$ uniformly on $[-1,1]$,

(3) for every sequence $\left\{t_{n}\right\}_{n \in \mathbb{N}}$ decreasing to zero the sequence of functions $\left\{f_{t_{n}}\right\}_{n \in \mathbb{N}}$ converges to $f(0)$ on $[-1,1]$,

(4) there exists a decreasing to zero sequence $\left\{t_{n}\right\}_{n \in \mathbb{N}}$ such that the sequence of functions $\left\{f_{t_{n}}\right\}_{n \in \mathbb{N}}$ converges to $f(0)$ uniformly on $[-1,1]$,

Key Words: Continuity, *-topology, Hashimoto topology, Density topology, I-density topology, Wilczyński topology, approximately and I-approximately continuous functions.

Mathematical Reviews subject classification: 26B05, 28A20, 54C30.

Received by the editors February 27, 1999 
(5) there are two points $x_{1} \in(-1,0)$ and $x_{2} \in(0,-1)$ such that for every decreasing to zero sequence $\left\{t_{n}\right\}_{n \in \mathbb{N}}$ the sequence of functions $\left\{f_{t_{n}}\right\}_{n \in \mathbb{N}}$ converges to $f(0)$ at $x_{1}$ and $x_{2}$.

Now we shall give several applications of this new approach to continuity with respect to the Hashimoto topology, density topology and $I$-density topology.

Let $\mathcal{S}$ be an algebra and $\mathcal{I}$ a proper ideal of subsets of the real line $\mathbb{R}$. We assume $\mathcal{S}$ and $\mathcal{I}$ to be invariant with respect to linear transformations. If $A \in \mathcal{S}$, we say that $A$ is $\mathcal{S}$-measurable. Similarly, if for a real function $f$, $f^{-1}(U)$ is $\mathcal{S}$-measurable for every open $U$, we say that $f$ is $\mathcal{S}$-measurable. If some property holds for points from $A \backslash P$ for some $P \in \mathcal{I}$, we say that it holds $\mathcal{I}$-almost everywhere ( $\mathcal{I}$-a.e.) on $A$. If $B=A \backslash P$ for some $P \in \mathcal{I}$, we say that $B$ is residual subset of $A$ or $B$ is residual in $A$. We use $A^{c}$ for the complement of $A$ and $A-x$ for $\{y-x: y \in A\}$.

For a given sequence $\left\{t_{n}\right\}_{n \in \mathbb{N}}$ of real numbers decreasing to zero we shall now consider the convergence of a sequence of functions $\left\{f_{t_{n}}\right\}_{n \in \mathbb{N}}$ on a given residual subset of $[-1,1]$.

Definition 1. We say that a sequence of functions $\left\{f_{n}\right\}_{n \in \mathbb{N}}$ converges a.e. uniformly on a set $A \subset \mathbb{R}$, if there is a residual subset $B$ of $A$ such that $\left\{f_{n}\right\}_{n \in \mathbb{N}}$ converges uniformly on $B$.

Theorem 2. Let $\mathcal{I}$ be a $\sigma$-ideal. For every real function $f$, if there exists a decreasing to zero sequence $\left\{t_{n}\right\}_{n \in \mathbb{N}}$ such that the sequence of functions $\left\{f_{t_{n}}\right\}_{n \in \mathbb{N}}$ converges to $f(0)$ a.e. uniformly on $[-1,1]$, then there exists a residual subset $E$ of $\mathbb{R}$ such that $f$ restricted to $E \cup\{0\}$ is continuous at 0 .

Proof. Let $A \in \mathcal{I}$ be the set such that $\left\{f_{t_{n}}\right\}_{n \in \mathbb{N}}$ converges to $f(0)$ uniformly on $[-1,1]-A$. Put $E=\mathbb{R} \backslash \bigcup_{n}\left(t_{n} \cdot A\right)$ and define a function $g$ such that

$$
g(x)= \begin{cases}f(x) & x \in E \\ f(0) & x \in \mathbb{R} \backslash E .\end{cases}
$$

Now the sequence $\left\{g_{t_{n}}\right\}_{n \in \mathbb{N}}$ converges uniformly to $f(0)$ on $[-1,1]$. By Theorem 1 (4) the function $g$ is continuous at 0 . Hence $g$ restricted to $E \cup\{0\}$ is continuous at 0 . As $g$ restricted to $E \cup\{0\}$ equals $f$ restricted to $E \cup\{0\}$, the proof is complete.

Theorem 3. Let $\mathcal{I}$ be a $\sigma$-ideal. For every real function $f$, if there exists a residual subset $E$ of $\mathbb{R}$ such that $f$ restricted to $E \cup\{0\}$ is continuous at 0 , then for every decreasing to zero sequence $\left\{t_{n}\right\}_{n \in \mathbb{N}}$ the sequence of functions $\left\{f_{t_{n}}\right\}_{n \in \mathbb{N}}$ converges to $f(0)$ a.e. uniformly on $[-1,1]$. 
Proof. Let $A=\mathbb{R} \backslash\{E \cup\{0\}\}$ and $B=\bigcup_{n}\left(\left(\frac{1}{t_{n}} \cdot A\right) \cap[-1,1]\right)$. As $E$ is residual, we have $B \in \mathcal{I}$. Define function $g$ such that

$$
g(x)= \begin{cases}f(x) & x \in E \cup\{0\} \\ f(0) & x \notin E \cup\{0\} .\end{cases}
$$

Clearly, $g$ is continuous at 0 , hence the sequence $\left\{g_{t_{n}}\right\}_{n \in \mathbb{N}}$ converges uniformly to $f(0)$ on $[-1,1]$. The sequence $\left\{f_{t_{n}}\right\}_{n \in \mathbb{N}}$ converges to $f(0)$ a.e. uniformly on $[-1,1]$, because $f_{n}$ restricted to $\mathbb{R} \backslash B$ equals $g_{n}$ restricted to $\mathbb{R} \backslash B$ for every $n \in \mathbb{N}$, and $B \in \mathcal{I}$.

Corollary 4. Let $\mathcal{I}$ be a $\sigma$-ideal. The following conditions are equivalent:

(1) there exists a residual subset $E$ of $\mathbb{R}$ such that $f$ restricted to $E \cup\{0\}$ is continuous at 0 ,

(2) there exists a decreasing to zero sequence $\left\{t_{n}\right\}_{n \in \mathbb{N}}$ such that the sequence of functions $\left\{f_{t_{n}}\right\}_{n \in \mathbb{N}}$ converges to $f(0)$ a.e. uniformly on $[-1,1]$,

(3) for every decreasing to zero sequence $\left\{t_{n}\right\}_{n \in \mathbb{N}}$ the sequence of functions $\left\{f_{t_{n}}\right\}_{n \in \mathbb{N}}$ converges to $f(0)$ a.e. uniformly on $[-1,1]$.

Remark 1. The above Corollary gives us a characterization of continuity at a point of a real function with respect to *topology (Hashimoto topology) generated by the basis $\{U \backslash P: U \in \tau, P \in \mathcal{I}\}$, where $\tau$ is a natural topology on $\mathbb{R}$ and $\mathcal{I}$ is a $\sigma$-ideal (see [3], [5]).

In 1982, W. Wilczyński in his paper [8] formulated the following definition:

Definition 2. We say that the point 0 is an $\mathcal{I}$-density point of an $\mathcal{S}$-measurable set $A$ if for any decreasing to zero sequence $\left\{t_{n}\right\}_{n \in \mathbb{N}}$ of real numbers there exists a subsequence $\left\{t_{n_{m}}\right\}_{m \in \mathbb{N}}$ such that $\chi_{\left\{\frac{1}{t_{n_{m}}} \cdot A\right\} \cap[-1,1]}$ converges to $1, \mathcal{I}$ almost everywhere on $[-1,1]$. (Equivalently such that $\liminf _{k \rightarrow \infty}\left(\left(\frac{1}{t_{n_{m}}} \cdot A\right) \cap\right.$ $[-1,1])=[-1,1] \backslash P$ for some $P \in \mathcal{I})$

We say that the point $x$ is an $\mathcal{I}$-density point of an $\mathcal{S}$-measurable set $A$ if 0 is an $\mathcal{I}$-density point of $A-x$.

If $\mathcal{S}$ is the $\sigma$-algebra of a Lebesgue measurable sets and $\mathcal{I}$ is the $\sigma$-ideal of sets of measure zero, this defines the usual density point of Lebesgue measurable set. If $\mathcal{S}$ is the $\sigma$-algebra of sets having Baire property and $\mathcal{I}$ is the $\sigma$-ideal of sets of the first category, this is a definition of an $\mathcal{I}$-density point of a Baire measurable set. The definition and all its consequences were deeply examined in a large number of papers - see [6], [7], and [1]. We adopt here their basic definitions. For $A \in \mathcal{S}$, let $\Phi(A)$ denote the set of all density 
points of $A$. The family $\{A \in \mathcal{S}, A \subset \Phi(A)\} \quad$ is a topology on $\mathbb{R}$ called the $\mathcal{I}$-density topology (Wilczyński topology) and is denoted $\tau_{\mathcal{I}}$. We say that $\mathcal{S}$ measurable function $f$ is topologically $\mathcal{I}$-approximate continuous at $x$, if and only if $f^{-1}(f(x)-a, f(x)+a)$ has $x$ as its density point, for every positive $a$. The continuity of real functions with respect to $\tau_{\mathcal{I}}$-topology was examined mainly for $\mathcal{S}$ the $\sigma$-algebra of a Lebesgue measurable sets and $\mathcal{I}$ the $\sigma$-ideal of sets of measure zero or $\mathcal{S}$ the $\sigma$-algebra $\mathcal{S}$ of Baire measurable sets with $\mathcal{I}$ the $\sigma$-ideal of sets of first category.

Remark 2. We say that an $\mathcal{S}$-measurable function $f$ is restrictively $\mathcal{I}$-approximate continuous at point $x$, if and only if there is a $\tau_{\mathcal{I}}$-open neighborhood $U$ of $x$ such that $f$ restricted to $U$ is continuous at $x$ in the natural topology relativised to $U$. The restrictional continuity of $\mathcal{S}$-measurable real function implies its topological continuity. In the case of $\mathcal{S}$ the $\sigma$-algebra of a Lebesgue measurable sets and $\mathcal{I}$ the $\sigma$-ideal of sets of measure zero, $\mathcal{I}$-approximate restrictional continuity and topological $\mathcal{I}$-approximate continuity coincide (See [4].)

Remark 3. Observe that the definition of an $\mathcal{I}$-density point can be reformulated in the following form: We say that the point 0 is an $\mathcal{I}$-density point of an $\mathcal{S}$-measurable set $A$ if for any sequence $\left\{t_{n}\right\}_{n \in \mathbb{N}}$ of real numbers decreasing to zero there exists a subsequence $\left\{t_{n_{m}}\right\}_{m \in \mathbb{N}}$ such that $\chi_{A}\left(t_{n_{m}} \cdot x\right)$ converges to $1, \mathcal{I}$-almost everywhere on $[-1,1]$.

This leads in the natural way to the following definition:

Definition 3. We say that an $\mathcal{S}$-measurable function $f$ has property $(*)$ at a point 0 , if and only if for any decreasing to zero sequence $\left\{t_{n}\right\}_{n \in \mathbb{N}}$ of real numbers there exists a subsequence $\left\{t_{n_{m}}\right\}_{m \in \mathbb{N}}$ such that $f\left(t_{n_{m}} \cdot x\right)$ converges to $f(0), \mathcal{I}$-almost everywhere on $[-1,1]$.

We say that an $\mathcal{S}$-measurable function $f$ has property $(*)$ at point $x$, if the function $g, g(y)=f(y-x)$, has property $(*)$ at 0 .

We shall show that for an $\mathcal{S}$-measurable function, the property $(*)$ at a point $x$ is equivalent to its $\mathcal{I}$-approximate topological continuity.

Theorem 5. If the $\mathcal{S}$-measurable function $f$ has property $(*)$ at a point $x$, then it is $\mathcal{I}$-approximate topologically continuous at this point.

Proof. We shall restrict ourselves to the case $x=0$ and additionally put $f(0)=0$. Suppose that $f$ is not $\mathcal{I}$-approximate topologically continuous at 0 . Then there exists $a>0$ such that the set $A=\{x:-a<f(x)<a\}$ has not 0 as its density point. It means that there exists a decreasing to zero 
sequence $\left\{t_{n}\right\}_{n \in \mathbb{N}}$ of real numbers such that for each subsequence $\left\{t_{n_{m}}\right\}_{m \in \mathbb{N}}$ the sequence $\chi_{\left\{\frac{1}{t_{n_{m}}} \cdot A\right\} \cap[-1,1]}$ does not converge to $1, \mathcal{I}$-almost everywhere on $[-1,1]$. This is equivalent to $\limsup _{k \rightarrow \infty}\left(\left(\frac{1}{t_{n_{m}}} \cdot A^{c}\right) \cap[-1,1]\right) \notin \mathcal{I}$.

For $x \in A^{c}$, we have $|f(x)| \geqslant a$, hence for $x \in \limsup _{k \rightarrow \infty}\left(\frac{1}{t_{n_{m}}} \cdot A^{c}\right)$ the sequence $f\left(t_{n_{m}} \cdot x\right)$ does not converge to $f(0)=0$. It follows that there exists a decreasing to zero sequence $\left\{t_{n}\right\}_{n \in \mathbb{N}}$ of real numbers such that for each subsequence $\left\{t_{n_{m}}\right\}_{m \in \mathbb{N}}$, the sequence $f\left(t_{n_{m}} \cdot x\right)$ does not converge to $f(0)=0$, I-a.e. on $[-1,1]$; this means that $f$ does not have property $(*)$ at the point 0 .

Theorem 6. Let $\mathcal{I}$ be a $\sigma$-ideal. If the $\mathcal{S}$-measurable function $f$ is $\mathcal{I}$-approximate topologically continuous at point $x$ then it has property $(*)$ at this point.

Proof. We restrict ourselves again to the case $x=0$ and additionally we put $f(0)=0$. As $f$ is $\mathcal{I}$-approximate topologically continuous at 0 then $A_{\varepsilon}=$ $f^{-1}((-\varepsilon, \varepsilon))$ has 0 as its density point, for every $\varepsilon>0$. The latter can be stated equivalently that 0 is a density point of $A_{k}=f^{-1}\left(\left(-\frac{1}{k}, \frac{1}{k}\right)\right)$, for every $k \in N$. Let $\left\{t_{n}\right\}_{n \in \mathbb{N}}$ be a sequence of real numbers decreasing to zero and put $k=1$. We may choose a subsequence $\left\{t_{n}^{(1)}\right\}_{n \in \mathbb{N}}$ of $\left\{t_{n}\right\}_{n \in \mathbb{N}}$ such that $\chi_{\left\{\frac{1}{t_{n}^{(1)}} \cdot A_{1}\right\} \cap[-1,1]}$ converges to $1, \mathcal{I}$-almost everywhere on $[-1,1]$. This means that there exists a set $E_{1}$ such that $[-1,1]-E_{1} \in \mathcal{I}$ and for points from $E_{1}$, $\chi_{\left\{\frac{1}{t_{n}^{(1)}} \cdot A_{1}\right\} \cap[-1,1]}$ converges to 1 . Since $E_{1}=\liminf _{m \rightarrow \infty}\left(\left\{\frac{1}{t_{n}^{(1)}} \cdot A_{1}\right\} \cap[-1,1]\right)$ we have $\limsup _{m \rightarrow \infty}\left|f\left(t_{n}^{(1)} \cdot x\right)\right|<1$ for $x \in E_{1}$. Similarly we can find, for every natural $k>1$, a set $E_{k} \subset E_{k-1}$ and a subsequence $\left\{t_{n}^{(k)}\right\}_{n \in \mathbb{N}}$ of $\left\{t_{n}^{(k-1)}\right\}_{n \in \mathbb{N}}$ such that $[-1,1] \backslash E_{k} \in \mathcal{I}$ and $\limsup _{m \rightarrow \infty}\left|f\left(t_{n}^{(k)} \cdot x\right)\right|<\frac{1}{k}$ for $x \in E_{k}$. Let $\left\{t_{n_{p}}\right\}_{p \in \mathbb{N}}$ be a diagonal subsequence of subsequences $\left\{t_{n}^{(k)}\right\}_{n \in \mathbb{N}}, k \in N$. We have $\limsup _{p \rightarrow \infty}\left|f\left(t_{n_{p}} \cdot x\right)\right|=0$ for $x$ from $\bigcap_{k=1}^{\infty} E_{k}$. Since $\mathcal{I}$ is a $\sigma$-ideal and $[-1,1] \backslash E_{k} \in \mathcal{I}$ for every $k \in N$ then $[-1,1] \backslash \bigcap_{k=1}^{\infty} E_{k} \in \mathcal{I}$. So, for any sequence $\left\{t_{n}\right\}_{n \in \mathbb{N}}$ of real numbers there exists a subsequence $\left\{t_{n_{p}}\right\}_{p \in \mathbb{N}}$ such that $f\left(t_{n_{p}} \cdot x\right)$ converges to $f(0), \mathcal{I}$-almost everywhere on $[-1,1]$, i.e., function $f$ has property $(*)$ at point 0 . 
If $\mathcal{I}$ is a $\sigma$-ideal, Theorems 5 and 6 state that for the $\mathcal{S}$-measurable function $f$ its $\mathcal{I}$-approximate topological continuity and property $(*)$ are equivalent at point $x$.

Theorem 7. If the $\mathcal{S}$-measurable function $f$ is $\mathcal{I}$-approximate restrictively continuous at point $x$ then it has property $(*)$ at this point.

Proof. Since the restrictional continuity implies the topological continuity, the theorem follows immediately. However, we would like to present the proof with the use of the property $(*)$. We shall restrict our considerations to the case $x=0$. From the assumption it follows that there exists an $\tau_{\mathcal{I}}$-open neighborhood $U$ of 0 such that $f$ restricted to $U$ is continuous at 0 . As 0 is an $\mathcal{I}$-density point of $U$, for any sequence $\left\{t_{n}\right\}_{n \in \mathbb{N}}$ of real numbers decreasing to zero there exists a subsequence $\left\{t_{n_{m}}\right\}_{m \in \mathbb{N}}$ such that $\chi_{\left\{\frac{1}{t_{n_{m}}} \cdot U\right\} \cap[-1,1]}$ converges to $1, \mathcal{I}$-almost everywhere on $[-1,1]$. This means that there exists a set $E$ such that $[-1,1]-E \in \mathcal{I}$ and for points from $E, \chi_{\left\{\frac{1}{\bar{t}_{m}} \cdot U\right\} \cap[-1,1]}$ converges to 1 . Put

$$
g(x)=\left\{\begin{array}{ll}
f(x) & x \in U \\
f(0) & x \notin U
\end{array} .\right.
$$

By the assumption $g$ is continuous at 0 and by Theorem $1(3), g\left(t_{n_{m}} \cdot x\right)$ converges to $f(0)$ everywhere on $[-1,1]$. Therefore, $f\left(t_{n_{m}} \cdot x\right)$ converges to $f(0)$ for points from $E$, i.e. $\mathcal{I}$-almost everywhere on $[-1,1]$.

Now we shall give a definition closely related to Lemma 1 in [7].

Definition 4. We shall say that the pair $(\mathcal{S}, \mathcal{I})$ is of type I (is of type II), if for every increasing sequences $\left\{t_{n}\right\}_{n \in \mathbb{N}},\left\{s_{n}\right\}_{n \in \mathbb{N}}$ of real numbers tending to infinity and such that

$$
\lim _{n \rightarrow \infty} \frac{t_{n}}{s_{n}}=1
$$

and for every $\mathcal{S}$-measurable set $A$ such that $\liminf _{n \rightarrow \infty}\left(t_{n} \cdot A\right) \cap[-1,1]$ is residual in $[-1,1]$ then also $\liminf _{n \rightarrow \infty}\left(s_{n} \cdot A\right) \cap[-1,1]$ is residual in $[-1,1]$ (there exists a subsequence $\left\{n_{m}\right\}_{m \in \mathbb{N}}$ such that $\liminf _{m \rightarrow \infty}\left(s_{n_{m}} \cdot A\right) \cap[-1,1]$ is residual in $\left.[-1,1]\right)$.

In Lemma 1 in [7] it is proved that the pair $(\mathcal{S}, \mathcal{I})$ where $\mathcal{S}$ is the $\sigma$-algebra of sets having Baire property and $\mathcal{I}$ the $\sigma$-ideal of sets of the first category is of type I. It is not difficult to show that in measure case the pair $(\mathcal{S}, \mathcal{I})$, where $\mathcal{S}$ is the $\sigma$-algebra of Lebesgue measurable sets and $\mathcal{I}$ the $\sigma$-ideal of sets of measure zero, is of type II. 
Lemma 8. Let $\mathcal{I}$ be a $\sigma$-ideal. Suppose a pair $(\mathcal{S}, \mathcal{I})$ is of type I (is of type II) and $\left\{t_{n}\right\}_{n \in \mathbb{N}},\left\{s_{n}\right\}_{n \in \mathbb{N}}$ are sequences of real numbers decreasing to zero such that

$$
\lim _{n \rightarrow \infty} \frac{t_{n}}{s_{n}}=1
$$

If for a real $\mathcal{S}$-measurable function $f, f\left(t_{n} \cdot x\right)$ converges to $f(0) \mathcal{I}$-almost everywhere on $[-1,1]$ then $f\left(s_{n} \cdot x\right)$ converges to $f(0) \mathcal{I}$-almost everywhere on $[-1,1]$. (There is a subsequence $\left\{s_{n_{m}}\right\}_{m \in \mathbb{N}}$ of $\left\{s_{n}\right\}_{n \in \mathbb{N}}$ such that $f\left(s_{n_{m}} \cdot x\right)$ converges to $f(0), \mathcal{I}$-a.e. on $[-1,1]$.)

Proof. Put $A_{k}=\left\{x \in[-1,1]:|f(x)|<\frac{1}{k}\right\}$. For every decreasing to zero sequence $\left\{u_{n}\right\}_{n \in \mathbb{N}}$ of real numbers we have

$$
\begin{aligned}
\bigcup_{p} \bigcap_{n \geqslant p}\left(\frac{1}{u_{n}} \cdot A_{k+1}\right) \cap[-1,1] & =\bigcup_{p} \bigcap_{n \geqslant p}\left\{x \in[-1,1]:\left|f\left(u_{n} \cdot x\right)\right|<\frac{1}{k+1}\right\} \\
& =\left\{x \in[-1,1]: \exists_{p} \forall_{n \geqslant p}\left|f\left(u_{n} \cdot x\right)\right|<\frac{1}{k+1}\right\} \\
& \subset\left\{x \in[-1,1]: \limsup _{n}\left|f\left(u_{n} \cdot x\right)\right| \leqslant \frac{1}{k+1}\right\} \\
& \subset\left\{x \in[-1,1]: \limsup _{n}\left|f\left(u_{n} \cdot x\right)\right|<\frac{1}{k}\right\} \\
& \subset\left\{x \in[-1,1]: \exists_{p} \forall_{n \geqslant p}\left|f\left(u_{n} \cdot x\right)\right|<\frac{1}{k}\right\} \\
& =\bigcup_{p} \bigcap_{n \geqslant p}\left\{x \in[-1,1]:\left|f\left(u_{n} \cdot x\right)\right|<\frac{1}{k}\right\} \\
& =\bigcup \bigcap_{p}\left(\frac{1}{u_{n}} \cdot A_{k}\right) \cap[-1,1] .
\end{aligned}
$$

Put $B_{k}=\bigcup_{p} \bigcap_{n \geqslant p}\left(\frac{1}{u_{n}} \cdot A_{k}\right) \cap[-1,1]$. Now we have

$$
\begin{gathered}
B_{k+1}=\bigcup_{p} \bigcap_{n \geqslant p}\left(\frac{1}{u_{n}} \cdot A_{k+1}\right) \cap[-1,1] \subset\left\{x \in[-1,1]: \limsup _{n}\left|f\left(u_{n} \cdot x\right)\right|<\frac{1}{k}\right\} \\
\subset \bigcup_{p} \bigcap_{n \geqslant p}\left(\frac{1}{u_{n}} \cdot A_{k}\right) \cap[-1,1]=B_{k}, \text { and }
\end{gathered}
$$




$$
\begin{aligned}
\bigcap_{k}\left\{x \in[-1,1]: \limsup _{n}\left|f\left(u_{n} \cdot x\right)\right|<\frac{1}{k}\right\} & =\bigcap_{k} \bigcup_{p} \bigcap_{n \geqslant p}\left(\frac{1}{u_{n}} \cdot A_{k}\right) \cap[-1,1] \\
& =\bigcap_{k} B_{k} .
\end{aligned}
$$

Hence,

$$
\begin{aligned}
& \left\{x \in[-1,1]: \lim _{n} f\left(u_{n} \cdot x\right)=0\right\}=\left\{x \in[-1,1]: \limsup _{n}\left|f\left(u_{n} \cdot x\right)\right|=0\right\} \\
= & \bigcap_{k}\left\{x \in[-1,1]: \limsup _{n}\left|f\left(u_{n} \cdot x\right)\right|<\frac{1}{k}\right\}=\bigcap_{k} \bigcup_{p} \bigcap_{n \geqslant p}\left(\frac{1}{u_{n}} \cdot A_{k}\right) \cap[-1,1] .
\end{aligned}
$$

By the assumption $f\left(t_{n} \cdot x\right)$ converges to $f(0), \mathcal{I}$-almost everywhere on $[-1,1]$, therefore $\left\{x \in[-1,1]: \lim _{n} f\left(t_{n} \cdot x\right)=0\right\}$ is residual on $[-1,1]$ and consequently $\bigcap_{k} \bigcup_{p} \bigcap_{n \geqslant p}\left(\frac{1}{t_{n}} \cdot A_{k}\right) \cap[-1,1]$ is residual on $[-1,1]$. As $\mathcal{I}$ is a $\sigma$-ideal, the countable intersection of sets is residual, if and only if every set is residual and the latter implies that $\bigcup_{p} \bigcap_{n \geqslant p}\left(\frac{1}{t_{n}} \cdot A_{k}\right) \cap[-1,1]$ is residual on $[-1,1]$, for every $k \in N$.

Now, if a pair $(\mathcal{S}, \mathcal{I})$ is of type I then also $\bigcup_{p} \bigcap_{n \geqslant p}\left(\frac{1}{s_{n}} \cdot A_{k}\right) \cap[-1,1]$ is residual on $[-1,1]$, for every $k \in N$ and $\bigcap_{k} \bigcup_{p} \bigcap_{n \geqslant p}\left(\frac{1}{s_{n}} \cdot A_{k}\right) \cap[-1,1]$ is residual on $[-1,1]$. By the last statement $\left\{x \in[-1,1]: \lim _{n} f\left(s_{n} \cdot x\right)=0\right\}$ is residual on $[-1,1]$ and $f\left(s_{n} \cdot x\right)$ converges to $f(0), \mathcal{I}$-almost everywhere on $[-1,1]$.

Suppose now that a pair $(\mathcal{S}, \mathcal{I})$ is of type II. Then for $k=1$ the set $\bigcup_{p} \bigcap_{n \geqslant p}\left(\frac{1}{t_{n}} \cdot A_{k}\right) \cap[-1,1]$ is residual on $[-1,1]$ and we may choose a subsequence $\left\{s_{n}^{(1)}\right\}_{n \in \mathbb{N}}$ of $\left\{s_{n}\right\}_{n \in \mathbb{N}}$ such that $\bigcup_{p} \bigcap_{n \geqslant p}\left(\frac{1}{s_{n}^{(1)}} \cdot A_{k}\right) \cap[-1,1]$ is residual on $[-1,1]$. For $k=2$, the set $\bigcup_{p} \bigcap_{n \geqslant p}\left(\frac{1}{t_{n}^{(1)}} \cdot A_{k}\right) \cap[-1,1]$ is residual on $[-1,1]$ and we may choose a subsequence $\left\{s_{n}^{(2)}\right\}_{n \in \mathbb{N}}$ of $\left\{s_{n}^{(1)}\right\}_{n \in \mathbb{N}}$ such that $\bigcup_{p} \bigcap_{n \geqslant p}\left(\frac{1}{s_{n}^{(2)}} \cdot A_{k}\right) \cap[-1,1]$ is residual on $[-1,1]$. Similarly, we can find for every natural $k>1$ a subsequence $\left\{s_{n}^{(k)}\right\}_{n \in \mathbb{N}}$ of $\left\{s_{n}^{(k-1)}\right\}_{n \in \mathbb{N}}$ such that $\bigcup_{p} \bigcap_{n \geqslant p}\left(\frac{1}{s_{n}^{(k)}} \cdot A_{k}\right) \cap[-1,1]$ is residual on $[-1,1]$. Let $\left\{s_{n_{m}}\right\}_{m \in \mathbb{N}}$ be a diagonal subsequence of subsequences $\left\{s_{n}^{(k)}\right\}_{n \in \mathbb{N}}, k \in \mathbb{N}$. The set $\bigcup_{p} \bigcap_{m \geqslant p}\left(\frac{1}{s_{n_{m}}} \cdot A_{k}\right) \cap$ 
$[-1,1]$ is residual on $[-1,1]$ for every $k \in \mathbb{N}$ so the set $\bigcap_{k} \bigcup_{p} \bigcap_{m \geqslant p}\left(\frac{1}{s_{n_{m}}} \cdot A_{k}\right) \cap$ $[-1,1]$ is also residual on $[-1,1]$ and $f\left(s_{n_{m}} \cdot x\right)$ converges to $f(0), \mathcal{I}$-a.e. on $[-1,1]$.

Theorem 9. Let $\mathcal{I}$ be a $\sigma$-ideal. Suppose a pair $(\mathcal{S}, \mathcal{I})$ is of type $I$ or II. If for the real $\mathcal{S}$-measurable function $f, f\left(s_{n} \cdot x\right)$ converges to $f(0) \mathcal{I}$-almost everywhere on $[-1,1]$ for some decreasing to zero sequence $\left\{s_{n}\right\}_{n \in \mathbb{N}}$ of real numbers such that $s_{k+1}>r \cdot s_{k}$, for some $r>0$ then $f$ has the property $(*)$ at 0 .

PROOF. Let $\left\{t_{n}\right\}_{n \in \mathbb{N}}$ be any sequence of real numbers decreasing to zero. Choose subsequences $\left\{s_{k_{p}}\right\}_{p \in \mathbb{N}}$ and $\left\{t_{n_{p}}\right\}_{p \in \mathbb{N}}$ such that $t_{n_{p}} \in\left(s_{k_{p}+1}, s_{k_{p}}\right]$. We have $0<r \cdot s_{k_{p}}<s_{k_{p}+1}<t_{n_{p}} \leqslant s_{k_{p}}$ and $0<r<\frac{t_{n_{p}}}{s_{k_{p}}} \leqslant 1$. We choose again subsequences $\left\{s_{k_{p_{m}}}\right\}_{m \in \mathbb{N}}$ and $\left\{t_{n_{p_{m}}}\right\}_{m \in \mathbb{N}}$ such that the sequence $\left\{\frac{t_{n_{p_{m}}}}{s_{k_{p_{m}}}}\right\}_{m \in \mathbb{N}}$ converges to some $a$, where $0<r \leqslant a \leqslant 1$. The sequence $\left\{\frac{t_{n_{p_{m}}}}{a \cdot s_{k_{p_{m}}}}\right\}_{m \in \mathbb{N}}$ converges to 1 and clearly $f\left(a \cdot s_{k_{p_{m}}} \cdot x\right)$ converges to $f(0), \mathcal{I}$-almost everywhere on $[-1,1]$. Directly from Lemma 8 there exists a subsequence $\left\{t_{n_{p_{m_{v}}}}\right\}_{v \in \mathbb{N}}$ of $\left\{t_{n_{p_{m}}}\right\}_{m \in \mathbb{N}}$ such that $f\left(t_{n_{p_{m_{v}}}} \cdot x\right)$ converges to $f(0), \mathcal{I}$-almost everywhere on $[-1,1]$. As $\left\{t_{n_{p_{m}}}\right\}_{v \in \mathbb{N}}$ is a subsequence of $\left\{t_{n}\right\}_{n \in \mathbb{N}}, f$ has the property $(*)$ at 0 .

Theorem 10. Let $\mathcal{S}$ be any algebra containing the Borel sets and $\mathcal{I}$ be an ideal. If $\left\{s_{n}\right\}_{n \in \mathbb{N}}$ is a sequence of real numbers decreasing to zero such that $\liminf _{n} \frac{s_{n+1}}{s_{n}}=0$, then there exists an $\mathcal{S}$-measurable function $f$ such that $f\left(s_{n} \cdot x\right)$

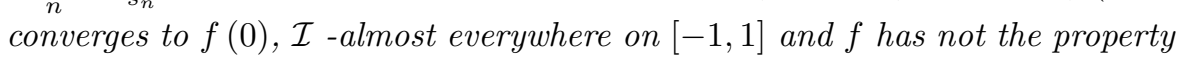
(*) at 0 .

Proof. The example from the necessary part of the Theorem 1 in [9] is good here.

As the consequence of Theorems 9 and 10, we have the following generalization of Theorem 1 in [9] and Theorem 1 in [2].

Corollary 11. Let $\mathcal{S}$ be any algebra containing the Borel sets and $\mathcal{I}$ a $\sigma$ ideal. Suppose a pair $(\mathcal{S}, \mathcal{I})$ is of type I or II. Then for every decreasing to zero sequence $\left\{s_{n}\right\}_{n \in \mathbb{N}}$ the condition that $s_{k+1}>r \cdot s_{k}$, for some $r>0$, is for every real function $f$ equivalent to the fact that $\mathcal{I}$-almost everywhere on $[-1,1]$ convergence of $f\left(s_{n} \cdot x\right)$ to $f(0)$ implies that $f$ has the property $(*)$ at 0. 


\section{References}

[1] K. Ciesielski, L. Larson, K. Ostaszewski, I-Density Continuous Functions, Mem. Amer. Math. Soc. 515(1994).

[2] J. Foran, Sequence conditions which imply approximate continuity, Real. Anal. Exchange 17(1991-92), 728-736.

[3] H. Hashimoto, On the *topology and its application, Fund. Math. 91(1976), 5-10.

[4] J. M. Jȩdrzejewski, On limit numbers of real function, Fund. Math. 83(1974), 269-281.

[5] D. Janković, R. Hamlett, New topologies from old via ideals, Amer. Math. Monthly, 97(4)1990.

[6] W. Poreda, E. Wagner-Bojakowska and W. Wilczyński, A category analogue of the density topology, Fund. Math. 125(1985), 167-173.

[7] W. Poreda, E. Wagner-Bojakowska and W. Wilczyński, Remarks on $\mathcal{I}$-density and $\mathcal{I}$-approximately continuous functions, Comm. Math. Univ. Carolinae 26(3)(1986), 263-277.

[8] W. Wilczyński, A generalization of the density topology, Real. Anal. Exchange 8(1)(1982-83), 16-20.

[9] W. Wilczyński, Sequence conditions which imply $\mathcal{I}$-approximate continuity, Tatra Mountains Math. Publ., 2(1993), 135-139. 